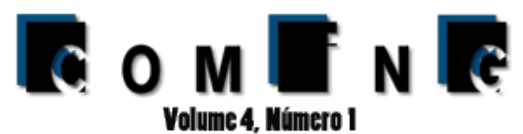

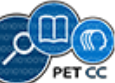

\section{Criação de Grupos de Pesquisa Discentes em cursos de Computação}

\author{
Gabriel Tobias Fuhr ${ }^{1}$, René Gargano Ferrari ${ }^{1}$, \\ Natan Luiz Paetzhold ${ }^{1}$, Giovani R. Librelotto ${ }^{1}$ \\ ${ }^{1}$ Curso de Ciência da Computação \\ Universidade Federal de Santa Maria (UFSM) - Santa Maria, RS - Brasil \\ \{gtfuhr,rgferrari,nlberwaldt, librelotto\}einf.ufsm.br
}

\begin{abstract}
In the academic path of students, it is not expected that all the academic subjects of their interest will be covered in the ordinary classes of their course. Therefore it is important that the students have a way to meet on themselves and to have contact with topics of their interest. This is possible with study groups, where students can share knowledge and motivate others students. This paper presents an analysis about a case study with undergraduates of Computer Science degree.
\end{abstract}

Resumo. Durante a trajetória acadêmica, os alunos nem sempre conseguem estudar todos os assuntos de seu interesse acadêmico, participando apenas das aulas previstas no currículo do seu curso. Devido a isso, é importante que os alunos tenham a alternativa de se reunir por conta própria para entrar em contato com áreas de conhecimento de seu interesse. Isso é possível através de grupos de estudo, onde alunos possam compartilhar conhecimento e motivar uns aos outros. Este artigo apresenta uma análise acerca de um estudo realizado com alunos do curso de Ciência da Computação.

\section{Introdução}

Durante a graduação, os alunos vivenciam muitas experiências, desafios e oportunidades que os preparam para suas futuras carreiras, seja para área de docência ou outros ramos do mercado de trabalho. Porém, como o tempo de um curso superior é limitado, haverá tópicos, muitas vezes importantes, que recebem pouca atenção ou sequer são abordados.

Para aqueles que buscam sucesso em suas carreiras, é de suma importância que tenham conhecimentos avançados em sua área de atuação, necessitando a busca de um saber além do que é ensinado em sala de aula. Aliadas à essa necessidade, existem muitas iniciativas por parte das universidades, como grupos de pesquisa orientados por um docente, programas de tutoria entre estudantes, ou até mesmo o estudo individual através de artigos científicos.

Porém, aqueles que decidem trilhar esse caminho através do estudo individual acabam por não evoluir seu conhecimento de forma tão eficiente quanto aqueles que se utilizam da troca de conhecimentos com outros alunos [Budi Yulianto 2016][Johnson 1999]. Por consequência, o aluno também deixará de exercitar suas soft skills [John 2009], as quais são pouco abordadas dentro do meio acadêmico. Ademais, segundo 
[Strandburg 2005], a cooperação é fundamental na construção do saber. Em virtude disso, este artigo tem como objetivo analisar e propor soluções para este tipo de problema, apresentando uma metodologia de estudo em grupo, vivenciada por alunos de Ciência da Computação da Universidade Federal de Santa Maria (UFSM).

O artigo está estruturado da seguinte forma: na Seção 2 citam-se trabalhos com estruturas similares às utilizadas nesta pesquisa, além de métodos para o desenvolvimento de um grupo de estudos.

A Seção 3, por sua vez, descreve a metodologia proposta para a criação de grupos de pesquisa discente em um ambiente universitário. Tal estratégia é definida com os seguintes passos: a definição do tema principal, que será abordado durante as reuniões do grupo; a definição do formato de encontro do grupo; as ferramentas que podem ser usadas para facilitar o contato entre os membros; a organização do grupo e gerenciamento de informações; colaborações externas que possam complementar a pesquisa.

$\mathrm{Na}$ Seção 4 apresenta-se um estudo de caso sobre a criação de um grupo de estudos sobre Machine Learning no curso de Ciência da Computação da UFSM. Esta seção aborda a estratégia utilizada na realização do estudo de caso.

A Seção 5 apresenta os resultados encontrados no estudo de caso. Por fim, na Seção 6 são apresentadas as considerações finais sobre a pesquisa. Com isso, esperase que o artigo consiga orientar estudantes durante a criação de grupos de estudo, que possam acrescentar conhecimento à sua formação acadêmica.

\section{Trabalhos Relacionados}

O modelo $3 \mathrm{C}$ de colaboração vem ao encontro como solução estrutural às necessidades de um grupo de estudos. Este modelo descreve quais são os papéis da comunicação, coordenação e colaboração em um ambiente colaborativo [Fuks 2008]. Além de demonstrar os papéis de tais ações, os autores também demonstram como as ferramentas de comunicação e de coordenação impulsionam o poder de colaboração de um grupo. Tais ensinamentos foram compartilhados em grupo, para incentivar a descoberta e o uso de tais ferramentas pelos participantes.

[John 2009] Trata do incentivo às habilidades interpessoais e também da utilização do modelo $3 \mathrm{C}$ e suas características. Como observado no trabalho citado anteriormente, na presente pesquisa os participantes do estudo de caso desenvolveram suas habilidades interpessoais, pois através do uso de ferramentas colaborativas e de encontros físicos e onlines puderam vivenciar experiências esperadas no mercado de trabalho [John 2009], soft skills. Desta forma, é visto que a atividade de estudar em grupo é socialmente prolífica e trará benefícios aos participantes. Além disso, um dos pilares do estudo em grupo, a cooperação, tem papel de protagonismo no aprendizado, segundo [Strandburg 2005]. O ato de debater em grupo sobre assuntos científicos também é importante para o desenvolvimento acadêmico, como visto em [Egilman 2013].

Para realçar a importância de se escolher um assunto interessante e relevante de uma área de conhecimento humano para o tema de um grupo de estudo, foi escolhido o trabalho de [Morgan 2018], o qual discute o modo disruptivo e inovador que o Machine Learning tem mudado diversas áreas. No tocante às subáreas do assunto escolhido pelo grupo, foi utilizado o [Russel d Ed], que discorre sobre os várias áreas complementares 
ao tema inteligência artificial.

Durante o caso de teste realizado neste artigo, foram utilizadas ferramentas digitais como meio de comunicação e aprendizado. Concluiu-se que tais ferramentas auxiliam no aprendizado dos discentes, porém não com a mesma eficiência do método de aprendizado presencial [Budi Yulianto 2016]. Em virtude disso, foi decidido mesclar ambas as metodologias de estudo, dessa forma, realizando-se uma reunião presencial semanal em conjunto à interações em plataformas colaborativas.

$\mathrm{O}$ interesse dos alunos na área do Machine Learning fez com que os mesmos precisassem buscar conhecimento no meio digital e também no meio acadêmico. Porém, não sendo suficiente tais compartilhamentos de saberes, surgiu a necessidade de um grupo de estudos. Somando-se a isso, a falta de pesquisas relacionadas a grupos de estudos, tornou útil o desenvolvimento da presente demonstração de uma estrutura de pesquisa, que obteve sucesso na sua criação. Espera-se que resultados deste artigo possam servir como incentivo para futuras iniciativas semelhantes em diferentes instituições.

\section{Criação de Grupos de Pesquisa Discente}

A presente seção trata da metodologia utilizada para a criação do clube de estudos, de forma que se permita utilizar o método de criação de grupos de estudo para qualquer área do conhecimento humano. Não se limitando a alunos do meio acadêmico, mas também a alunos de outros níveis de aprendizagem.

A seção foi dividida em quatro subseções. A subseção 3.1 descreve a definição do tema do grupo, demonstrando a importância do debate, entre os alunos interessados, sobre a definição do assunto a ser investigado. A seção 3.2 define o formato de encontros do grupo e a forma de organização utilizada na estrutura desses encontros. A subseção 3.3 apresenta a organização do grupo e gerenciamento de informações, como a importância de utilizar diferentes ferramentas para uma melhor comunicação e coordenação do clube. A subseção 3.4 mostra os benefícios de uma parceria com uma organização de pesquisa já estabelecida no ambiente acadêmico.

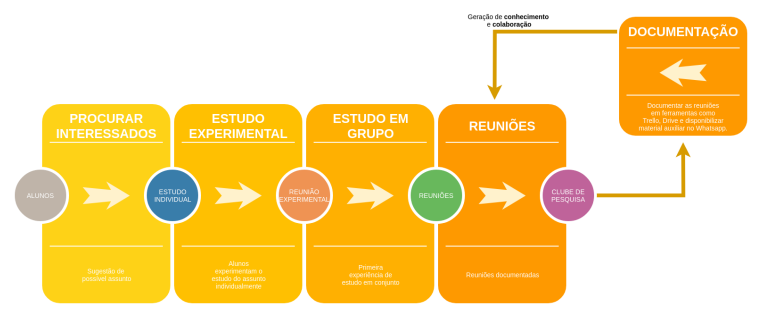

Figura 1. Um fluxograma que demonstra os passos descritos a seguir.

\subsection{Definição do tema do Grupo}

Conforme referido anteriormente, durante as aulas de um curso específico, surgem necessidade de aprofundar conhecimentos em determinados temas ou áreas de pesquisa. Essa investigação é causada pelas necessidades do mercado de trabalho, para resolver problemas sociais ou para o desenvolvimento de novas teorias acerca de um determinado tema. Nem sempre os alunos conseguem aprofundar seu conhecimento sobre assuntos 
de seu interesse acadêmico. Assim, a necessidade da pesquisa surge por diferentes motivos, por isso a necessidade do debate acerca do tema a ser estudado. De acordo com [Egilman 2013], o debate torna-se necessário para que todos os participantes estejam estimulados a pesquisa.

Para a definição real do tema a ser estudado pelo grupo, é necessário que todos tenham um interesse em comum, o qual incentiva os membros a buscar novos conteúdos, procurar desafios, pesquisar por possíveis inovações na área e trazer destaques para o seu futuro currículo. Em [Strandburg 2005] afirma-se que, o principal critério para que se realize a escolha de um tema, seria o que os alunos tenham por ele como um interesse mútuo, aumentando o engajamento nas atividades do grupo.

Vale ressaltar, que a formação de grupos de pesquisa produz um comportamento de independência nos alunos em relação à matriz curricular. Esse comportamento proporciona a escolha, sem nenhum viés, relacionado a estrutura e organização do curso. $\mathrm{O}$ fato de um assunto já ser contemplado pela ementa de alguma disciplina não impede os alunos de tomarem o mesmo como tema do grupo, pois o assunto pode ser de interesse dos alunos, mas limitado pelo tempo avaliável ao professor em sala de aula.

Incentiva-se a escolha em grupo do assunto, obtendo-se, assim, uma definição verdadeiramente colaborativa. Ferramentas que auxiliam no processo destas escolhas colaborativas e votações foram criadas e são usadas no processo de decisões no meio acadêmico e profissional. O Doodle ${ }^{1}$ seria um exemplo de ferramenta que auxilia no processo de escolhas e decisões realizadas, a mesma disponibiliza a votação de forma acessível em uma plataforma online. Recomenda-se também o uso da plataforma nos momentos de escolhas de datas para as reuniões do grupo.

\subsection{Definição do formato de encontro do grupo}

As plataformas virtuais representam um importante recurso para a realização de pesquisa em grupos. É importante salientar, entretanto, que, para a efetiva troca de ideias e de experiências entre os participantes, é necessário o contato direto, físico. Para que isso ocorra torna-se necessário delimitar um local de fácil acesso a todos, garantindo a maior participação possível. Para essa reunião de informações, vários meios podem se fazer úteis, mas para a eficiência do grupo de estudos, deve ocorrer o contato direto entre os membros. Através de reuniões presenciais, com um conteúdo previamente programado, os participantes se organizam e delimitam tarefas em um ambiente favorável ao estudo e ao compartilhamento de informações.

A delimitação do horário dos encontros deve ser programada de acordo com a demanda. Não são indicadas reuniões muito longas, somente o tempo suficiente para que o grupo se organize, mas não se disperse em outros assuntos durante o encontro. Deve-se contar com a duração adequada para que seja possível resolver todos os tópicos estabelecidos. Caso a reunião presencial não seja possível, ferramentas de comunicação, como mensageiros instantâneos ou videoconferência são alternativas para que as reuniões do grupo sejam realizadas.

Uma metodologia muito utilizada em projetos e que também pode ser aplicada em grupos de estudo é o SCRUM. A mesma foi apresentada em [Géron 2017] e resume-se

\footnotetext{
${ }^{1}$ Www.doodle.com
} 


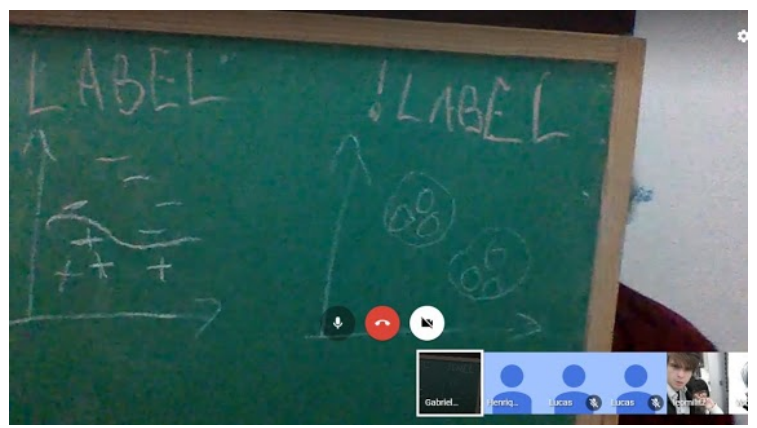

Figura 2. Exemplo de reunião realizada de forma online durante o estudo de caso realizado.

em uma forma ágil e eficaz para a realização de um projeto. As ações a serem realizadas em um projeto são organizadas em uma lista que é conhecida como Product Backlog. No início de cada Sprint, faz-se um Sprint Planning Meeting, ou seja, uma reunião de planejamento na qual o Product Owner prioriza os itens do Product Backlog e a equipe seleciona as atividades que ela será capaz de implementar durante o Sprint que se inicia. As tarefas alocadas em um Sprint são transferidas do Product Backlog para o Sprint Backlog.

No SCRUM, os projetos são divididos em ciclos, normalmente mensais, chamados de Sprints. Para aplicá-lo é necessário que haja um scrum master, geralmente o líder do grupo, que irá dividir o projeto em várias etapas, comumente chamadas de sprints. Cada sprint tem várias tarefas que precisam ser finalizadas até o prazo definido pelo scrum master. Durante o período da sprint, sempre que houver reuniões, cada membro do projeto deve resumir brevemente como está o andamento da sua tarefa atual. Desse modo há transparência na realização das tarefas e os membros podem ajudar, caso notem que algum colega precise. Sempre que uma tarefa for concluída deve-se enviá-la para ser revisada pelo scrum master. Quando todas as tarefas forem finalizadas é feita uma nova reunião para revisar todas as tarefas realizadas e decidir que tarefas farão parte do próximo sprint.

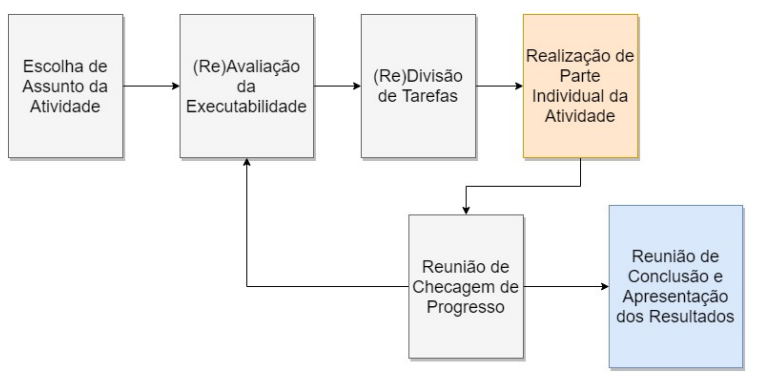

Figura 3. Um fluxograma que demonstra o funcionamento da metodologia ágil scrum.

A cada dia de uma Sprint, a equipe faz uma breve reunião, chamada Daily Scrum. O objetivo é disseminar conhecimento sobre o que foi feito anteriormente, identificar impedimentos e priorizar o trabalho posterior.

Ao final de um Sprint, a equipe apresenta as funcionalidades implementadas em uma Sprint Review Meeting. Finalmente, faz-se uma Sprint Retrospective e a equipe parte para o planejamento do próximo Sprin. Assim reinicia-se o ciclo. Veja a ilustração abaixo: 


\subsection{Organização do grupo e gerenciamento de informações}

O grupo, com a intenção de criar um ambiente colaborativo para seus integrantes, deve ter e criar ferramentas para facilitar a coordenação, colaboração e comunicação em grupo, conforme observado no modelo de colaboração 3C, apresentado em [Fuks 2008]. Tais ferramentas geralmente encontram-se disponíveis forma gratuita para a utilização em meios acadêmicos e uso pessoal [Budi Yulianto 2016]. Várias ferramentas foram experimentadas no caso de estudo. Dentre estas, as que mais possibilitaram um aumento na capacidade de comunicação e interação entre o grupo foram o Trello $^{2}$, divisão de tarefas e coordenação dos projetos do grupo, e o Whatsapp ${ }^{3}$, que se mostrou versátil e prático para discussões breves sobre assuntos do clube.

No quesito de banco de dados do clube, observou-se no caso de estudo a praticidade da plataforma Google Drive $e^{4}$ durante o compartilhamento de artigos, livros no formato digital e atas das reuniões realizadas. Vale lembrar também sobre o Doodle para a realização de votações e de escolhas de datas para encontros e realização de projetos.

Estas e outras ferramentas oferecem a possibilidade de catalisar uma rede de colaboração de diferentes clubes de estudos, pois independem do assunto de dados para o seu funcionamento. Com isso, recomenda-se a utilização de ferramentas como as citadas anteriormente durante a organização e coordenação do grupo.

\subsection{Parcerias externas}

Uma vez o grupo reunido e pronto para atuar, diversas dificuldades podem aparecer, como a baixa produtividade e a falta de embasamento, necessários a fundamentação, na investigação dos fatos. Algumas delas podem acabar tornando-se sérios obstáculos para o devido funcionamento do grupo. Nestas horas, a busca por apoio externo pode ser a melhor alternativa; grupos de pesquisa locais, professores, coordenação do(s) curso(s), Programas de Ensino Tutorial, todos podem tornar-se bons parceiros em momentos de dificuldade.

Um exemplo bastante notável é a necessidade de uma sala para que sejam feitas reuniões presenciais do grupo, muitas vezes é possível que os alunos não tenham à disposição um ambiente que favoreça a prática. Desta forma, um meio de cruzar este obstáculo é procurar um grupo institucional existente, que possua acesso a uma sala, e que possa ser cedida para os encontros.

Outra situação que pode surgir é aquela em que o grupo chega em tal ponto da curva de aprendizado no qual não consegue progredir, seja pelo conteúdo não estar disponível de forma clara nas bases de referência usadas pelo grupo, ou por haver uma encruzilhada, onde o conteúdo ou objeto de estudo se divide em múltiplos subassuntos e não há concordância entre os membros para onde seguir. Nessa hora o auxílio externo se faz imprescindível, este devendo ser buscado com um especialista na área, que possa palestrar o tópico de dúvida ao grupo e elucidar o caminho a ser seguido.

\footnotetext{
${ }^{2}$ https://trello.com/

${ }^{3}$ https://www.whatsapp.com/

${ }^{4} \mathrm{https}$ ://www.google.com.br/drive/apps.html
} 


\section{Caso de Estudo: Grupo de Pesquisa em Machine Learning}

Esta seção descreve os resultados obtidos pelos alunos do grupo, quese organizaram para dominar e ensinar diferentes técnicas de aprendizado de máquina [Géron 2017].

O clube do Machine Learning, foi criado através do interesse mútuo dos primeiros participantes em sua área de foco, o Aprendizado de Máquina. O interesse comum pelo tema foi identificado através de discussões iniciais, realizadas durante as pausas das aulas do curso. Tais momentos serviram como um exemplo de um tempo destinado à capacidade de ampliar conhecimentos afins às temáticas contempladas na grade curricular. Pode-se observar que, conceitos normalmente considerados complexos, quando enfrentados em grupo e com cooperação, acabam se tornando mais fáceis. Apesar do enfoque em aprendizado de máquina, assuntos relacionados como coleta, tratamento de dados, e padrões de projetos da área e avaliação de desempenho das aplicações também foram discutidas.

Muitos alunos também acabaram vendo no clube uma forma de criar conexões com pessoas que possuem interesses em comum, criando assim uma rede de alunos interessadas em explorar novas áreas, conteúdos e desenvolver soft skills, o que pode ser de grande valia no desenvolvimento dos envolvidos, não apenas no clube, mas como também em sua futura vida profissional e acadêmica.

Soft skills são habilidades comportamentais que auxiliam nas relações interpessoais entre colegas de trabalho ou até mesmo de estudos. Como mostrado em [John 2009], tais habilidades estão sendo valorizadas no mercado de trabalho atual. Ainda segundo [John 2009], artigos, a exposição regular de saberes, auxilia na sua apropriação pelos estudantes soft skills, ou seja, os mesmo apresentam um aumento no domínio desse conhecimento.

O exercício que incentiva a cooperação entre os alunos do curso aumenta o seu potencial para participar de projetos mais amplos. Pois tais projetos contarão com equipes maiores e uma das grandes habilidades esperadas pelo profissional atual é o trabalho em equipe. Portanto, além de potencializar e incentivar o desenvolvimento técnico de seus integrantes, o clube também tem o papel de preparar seus membros para a gerência de equipes em projetos da área.

As reuniões acabaram se formando a partir da extração do conceito principal das discussões feitas de maneira informal, o diálogo entre os participantes e a troca de experiências, tal formato de reunião foi se definindo de maneira mais organizada de acordo com o decorrer de novos encontros.

Mostrou-se necessário também a iniciativa de um dos membros para planejar, mediar as atividades do grupo e gerar assuntos base para os encontros do clube, pois foi observado que os alunos possuíam uma disponibilidade de tempo escassa para trazer novos assuntos de maneira constante a cada reunião. Graças ao hiato de novas tecnologias, $o$ grupo demonstrou baixa no interesse. $\mathrm{O}$ membro que assumir a responsabilidade torna-se responsável para identificar a fonte do problema do grupo. Caso o problema seja a falta de motivação causada por demasiada apresentação de conteúdos teóricos, uma das soluções observadas foi a de desenvolver projetos práticos de aprendizado e coordenar de maneira igualitária e de acordo com as habilidades específicas de cada participante a distribuição de tarefas entre os membros do clube. 


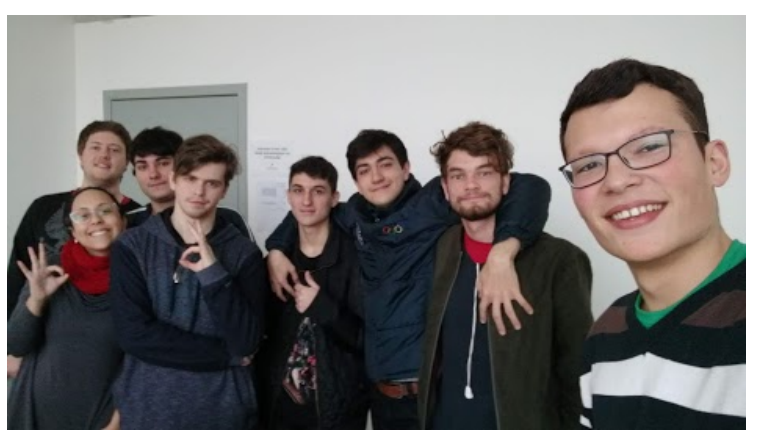

Figura 4. Integrantes do clube reunidos após uma reunião realizada de forma presencial durante o estudo de caso.

\subsection{Tema escolhido: Machine Learning}

O Machine Learning, ramo da Inteligência Artificial, permite que seja possível extrair importantes informações e padrões de dados como imagens, documentos, vídeos e tabelas [Géron 2017]. As técnicas usadas estão se tornando viáveis graças ao grande aporte de dados que os usuários da internet vêm fornecendo, tudo isso tem trazido soluções inovadoras para a sociedade. Tais soluções podem ser utilizadas em tarefas como o reconhecimento de voz, compra e venda de ações, desenvolvimento de carros autônomos e até mesmo diagnósticos de saúde mais precisos.

Soluções revolucionárias como essas entraram em consonância com a curiosidade de oito discentes de Ciência da Computação, que ficaram entusiasmados com a ideia de fazer com que o computador encontre uma forma de resolver problemas que não possuem resolução algorítmica. Pensando em estimular a capacidade de descoberta de cada um, em grupo, a ideia de um clube do Machine Learning veio à tona.

A grade curricular do curso de Ciência da Computação oferece uma cadeira de Inteligência Artificial no sétimo semestre, penúltimo semestre do curso. Sua ementa conta com uma abordagem ampla da grande área de Inteligência Artificial, que engloba várias subáreas como lógica, probabilidade, estatística, percepção, raciocínio e aprendizado.

[Russel d Ed] o que torna o enfoque em Aprendizado de Máquina algo irreal em apenas um semestre da disciplina. Vale ressaltar, que conceitos relacionados apresentados em diferentes cadeiras ajudam a aumentar o interesse de alunos no ramo de Inteligência Artificial. Destas podem-se citar as disciplinas que trabalham o paradigma lógico da Computação, como por exemplo Paradigmas da Programação e Lógica de Predicado. Ambas em parte abordando o estudo de métodos para se traduzir ideias e sentenças geradas na linguagem humana para predicados, estrutura sintática da programação lógica, para que possam ser interpretadas pela máquina. Mesmo com o fomento do interesse na área de Inteligência Artificial, os discentes carecem de uma disciplina com enfoque em Aprendizado de máquina no decorrer da graduação.

A Universidade Federal de Santa Maria também conta com a disciplina de Aprendizado de Máquina, sendo oferecida apenas como uma matéria do Programa de Pós Graduação em Ciência da Computação. Apesar disso, um discente da graduação pode cursá-la, caso atenda diversos requisitos da categoria Aluno Especial de Pós-Graduação, como por exemplo: possuir $75 \%$ dos créditos necessários para a conclusão do seu curso e 
a participação de projetos de pesquisa aprovados na instituição. Como pode-se observar, acessar os conhecimentos desta área de conhecimento, em crescimento, não é possível para quem está na fase inicial de seu curso de graduação.

O Machine Learning, uma área em rápida expansão e crescimento [Morgan 2018], está tendo inúmeros avanços graças a várias áreas da Computação e Matemática que foram criando uma forte fundamentação teórica para as suas técnicas de prospecção de dados. Visto isso, os alunos devem ter saber consolidado para produzir conhecimento de qualidade durante a sua contribuição em conjunto ao clube. Tais áreas da matemática e computação envolvem Álgebra Linear, Probabilidade, Fundamentos de Banco de Dados e Noções de programação. Porém, o grupo é formado por alunos de diversos graus de conhecimento, tais graus de conhecimento sendo definidos como a progressão nos semestres do curso, o que faz com que a cooperação entre participantes mais experientes e novos no curso seja necessária e incentivada.

O elevado número de notícias e pesquisas na área despertou o interesse dos discentes do curso. Também como oportunidades de empregos e crescimento de vagas no exterior no ramo de Machine Learning. Este crescimento se deve ao grande leque de possibilidades a serem exploradas através das técnicas de Machine Learning, tal capacidade é mostrada na pesquisa "Artificial Intelligence and Economic Growth" [Jones 2017] do Bureau Nacional de Pesquisa Econômica dos Estados Unidos.

Para se modificar a grade curricular de um curso superior, o seu corpo administrativo deve fazer um estudo das mudanças necessárias, além de vários passos burocráticos a serem realizados pela coordenação do mesmo, tal processo pode ser demorado. Por outro lado, as novas tecnologias e tendências surgem de maneira ágil, pensando nisso, a escolha de um assunto de crescimento recente, como o Aprendizado de Máquina no exemplo prático apresentado, que é pouco explorado pela grade curricular padrão do curso, estimula o interesse e o engajamento de possíveis membros ao clube, visto que os mesmos terão acesso antecipado a tecnologias inovadoras enquanto a grade curricular do curso não o integra.

O Curso de Ciência da Computação, apesar de bastante abrangente, não aborda alguns pontos com profundidade, como a linguagem Python e suas utilidades. Com a abertura do clube, discentes de todos os semestres do curso tiveram a oportunidade de entrar em contato com a linguagem e suas funcionalidades. Junto a ela foram abordadas várias bibliotecas como pandas, matplotlib, scikit-learn e numpy. Além disso, ferramentas como a interface Anaconda Navigator e o site https://www.kaggle.com/ foram utilizados como facilitadores no aprendizado e desenvolvimento de algoritmos de machine learning.

Uma vez por semana ministra-se o encontro do clube, o qual busca, através da metodologia utilizada, trazer o máximo de interatividade entre os membros. Foi sugerido aos alunos integrantes do clube que toda semana trouxessem assuntos, inovações, vídeos, livros, artigos e curiosidades relacionadas ao mundo do aprendizado de máquinas.

O clube foi criado de forma independente da intervenção direta dos docentes do curso, o que deu aos alunos mais liberdade na área de gerência das atividades, porém nada impede a conexão de alunos e professores para que novas oportunidades sejam abertas aos alunos. Devido a isso, foram utilizadas ferramentas que não possuíam vínculo 
com a instituição como o Google Drive ${ }^{5}$, Whatsapp ${ }^{6}$, Doodle $^{7}$ e Trello ${ }^{8}$ como parte da metodologia para organização das tarefas do clube.

Um ponto forte em favor da reunião de um grupo independente para o aprendizado de um assunto em contrapartida ao aprendizado em sala de aula, é a possibilidade de implementação de diversas atividades com caráter lúdico para incentivo ao estudo, podendo citar neste caso, o desenvolvimento de autômatos virtuais capazes de aprender a simular interpretação e ação humana em jogos eletrônicos, sendo capazes de desenvolver perícia na solução de tarefas propostas pelo jogo. Com o uso destes recursos é possível se ter uma visão bastante palpável do que se está trabalhando, aproximando o estudo do que é visto no dia-a-dia de muitos alunos.

Um exemplo disso é a pilotagem de um veículo sobre um mapa virtual: uma vez que a máquina tenha aprendido a se comportar de maneira aceitável como um suposto piloto humano neste espaço simulado, indo de um ponto a outro do cenário no menor tempo possível sem cometer infrações de trânsito. O que se tem é basicamente uma versão simplificada da tecnologia de veículos autônomos, que está cada vez mais próxima de se tornar parte do cotidiano das pessoas.

Entretanto, apesar da independência do grupo em relação à participação de professores, ainda assim recebeu-se apoio externo incentivando suas práticas, seja por parte do Programa de Ensino Tutorial, o qual deu incentivo inicial e ideias sobre a organização. Ainda, o Núcleo de Ciência da Computação favoreceu com a disponibilização de diferentes salas de estudo para uso de todos os alunos do curso, podendo o grupo fazer uso de uma delas para suas reuniões semanais.

\section{Discussão}

Ao concluir o primeiro semestre do projeto, pode-se observar pontos a melhorar no próximo, como a metodologia utilizada para compartilhar conhecimento entre os integrantes e a coordenação na hora da distribuição de tarefas entre os membros do clube. Ademais, vale ressaltar, que a coordenação do Curso de Ciência da Computação aprovou a iniciativa dos alunos envolvidos, através do registro da atividade no Sistema Acadêmico. Percebe-se uma integração e o interesse dos membros pela participação nas reuniões até mesmo em dias não letivos através de ferramentas de videoconferência.

Neste princípio de criação do grupo de pesquisa discente, trabalhou-se com uma amostra de 8 alunos, dos quais todos já eram familiarizados com lógica e programação, porém nem todos tinham experiência com a linguagem Python e com Machine Learning. Como o clube era feito puramente de alunos interessados no assunto e não exigia presença ou participação, é natural que nem todos mostrassem a mesma proatividade e engajamento. Ao final do primeiro semestre, todos se mostraram dispostos a continuar participando do Clube.

O método de abordagem dos tópicos foi bem aceito pelos participantes do clube. A prática inicial foi conversar sobre os métodos básicos de programação em Machine

\footnotetext{
${ }^{5}$ https://www.google.com.br/drive/apps.html

${ }^{6}$ https://www.whatsapp.com/

${ }^{7}$ https://doodle.com/

${ }^{8}$ https://trello.com/
} 
Learning, discutindo livros sobre o assunto e apresentando as ferramentas básicas geralmente utilizadas para se lidar com o assunto em questão. Após a introdução dos alunos ao assunto, foi sugerido aos mesmos que trouxessem notícias, vídeos e artigos que os interessassem. Quando todos já tinham adquirido certa experiência no assunto, foi feita a tentativa de realizar um projeto prático de Machine Learning. Esse foi o único método que não obteve bons resultados, pois os alunos não obtiveram sucesso em finalizar a proposta, acredita-se que por ter sido introduzida muito cedo ou em época em que os alunos estavam envolvidos em muitas provas e trabalhos.

Ao se iniciar o segundo semestre do projeto, tornou-se necessário ressaltar os pontos a melhorar levantados no último semestre, como a metodologia utilizada para compartilhar conhecimento entre os integrantes e a dinâmica utilizada na distribuição de tarefas entre os mesmos. Ademais, o clube despertou o interesse de outros alunos do curso, incluindo calouros, totalizando 11 integrantes na primeira reunião do primeiro semestre de 2019. Tais alunos terão de ser recebidos com uma atenção especial pelos integrantes, visto que podem carecer de uma base teórica, com isso as reuniões iniciais foram focadas em revisar a fundação teórica e computacional da área.

Mostrando a multidisciplinaridade das aplicações do Machine Learning, o clube recebeu propostas através de professores do curso, sobre projetos realizados em outros cursos que necessitam de implementações de Machine Learning. Visto isso, se espera que um resultado positivo seja gerado na vida Acadêmica dos participantes do clube no ano de 2019.

\section{Conclusão}

O objetivo principal deste artigo é o de guiar alunos na criação de um grupo de estudos independente, ou seja, sem a necessária intervenção de docentes. O estudo de caso descrito acompanhou e analisou a jornada de alunos de Ciência da Computação na criação de um clube focado em aprendizado de máquinas. O artigo também aborda a escolha do assunto do clube, metodologia utilizada nos encontros, ferramentas usadas para armazenar informações importantes das reuniões e a documentação do processo de aprendizagem e engajamento desses discentes.

Os resultados, após findado o primeiro semestre, foram satisfatórios e, dentro do esperado, notou-se grande evolução dos alunos envolvidos na área de atuação escolhida para debate dentro do grupo. Um dos motivos que assistiu o desenvolvimento dos alunos foi a iniciativa de um deles em tomar a liderança do grupo, ministrando as reuniões, levantando tópicos a serem debatidos e registrando em atas todos os encontros feitos. O papel do líder mostrou-se importante, pois muitas vezes os alunos não dispunham de tempo fora do horário de reunião para pesquisarem assuntos a serem debatidos. Além disso, notou-se que a reunião presencial foi o método de encontro que resultou em melhor desempenho e aprendizado dos membros. Tais encontros foram complementados com o uso de ferramentas que auxiliaram o grupo a manter contato e armazenar dados fora das reuniões como, por exemplo: WhatsApp ${ }^{9}$, Google Drive ${ }^{10}$ Trello $^{11}$ e Discord ${ }^{12}$.

\footnotetext{
${ }^{9}$ https://www.whatsapp.com/

${ }^{10} \mathrm{https}: / /$ www.google.com.br/drive/apps.html

${ }^{11} \mathrm{https} / / /$ trello.com/

${ }^{12} \mathrm{https} / / /$ discordapp.com/
} 
Por fim, conclui-se que o método de estudos através de um grupo, se reunindo e debatendo ideias e práticas, é bastante eficiente. Torna-se proveitoso para todos os participantes que se empenharem efetivamente com o projeto. Pode contribuir, ainda, compartilhando e aplicando a meios externos os conhecimentos adquiridos durante os trabalhos. Uma experiência como esta, em que um grupo de estudos constrói o conhecimento através de métodos pró-ativos e independentes, prepara os integrantes para projetos mais avançados e complexos.

\section{Referências}

Budi Yulianto, Harjanto Prabowo, R. R. K. (2016). Comparing the effectiveness of digital contents for improving learning outcomes in computer programming for autodidact students. Journal of e-Learning and Knowledge Society.

Egilman, D. S. (2013). The importance of scientific debate. International Journal of Occupational and Environmental Health.

Fuks, Hugo. Raposo, A. G. M. A. P. M. L. C. J. P. (2008). The 3c collaboration model. Encyclopedia of E-Collaboration.

Géron, A. (2017). Hands-On Machine Learning with Scikit-Learn \& Tensorflow. O’Reilly Media, Inc.

John, D. J. (2009). Study on the nature of impact of soft skills training programme on the soft skills development of management students. Pacific Business Review.

Johnson, David W. Johnson, R. T. (1999). Making cooperative learning work. THEORY INTO PRACTICE of The Ohio State University, Volume 38.

Jones, Charles I. Aghion, P. J. B. F. (2017). Artificial intelligence and economic growt. Institute for Economic Policy Research (SIEPR).

Li, M. P. Lam, B. H. (2005). Cooperative learning. The Active Classroom, The Hong Kong Institute of Education.

Morgan, P. (2018). Machine Learning Is Changing the Rules. O'Reilly Media, Inc.

Russel, Stuart. Norvig, P. (2009 (3rd Ed.)). Artificial Intelligence. A Modern Approach. Prentice Hall, New Jersey, United States.

Strandburg, K. J. (2005). Curiosity-driven research and university technology transfer. University Entrepreneurship and Technology Transfer.

Sutherland, J. J. S. (2014). Scrum The Art of Doing Twice the Work in Half the Time. Currency, Redfern, New South Wales. 